\title{
Randomised trial comparing biweekly oxaliplatin plus oral capecitabine versus oxaliplatin plus i.v. bolus fluorouracil/ leucovorin in metastatic colorectal cancer patients: results of the Southern Italy Cooperative Oncology study 0401
}

\author{
Pasquale Comella $\cdot$ Bruno Massidda $\cdot$ Gianfranco Filippelli $\cdot$ Antonio Farris $\cdot$ Donato Natale $\cdot$ Giuseppe Barberis \\ Luigi Maiorino $\cdot$ Sergio Palmeri $\cdot$ Michele Cannone $\cdot$ Giovanni Condemi $\cdot$ on behalf of the Southern Italy \\ Cooperative Oncology Group
}

Received: 26 March 2008 / Accepted: 5 August 2008 / Published online: 22 August 2008

(C) Springer-Verlag 2008

\begin{abstract}
Purpose Oxaliplatin combined with either fluorouracil/ leucovorin (OXAFAFU) or capecitabine (OXXEL) has a demonstrated activity in metastatic colorectal cancer patients. We aimed at comparing these two regimens in terms of response rate (RR), safety, progression-free survival (PFS), and quality of life (QoL) of patients.

Methods A total of 322 patients with metastatic colorectal cancer were randomized to receive biweekly: oxaliplatin $100 \mathrm{mg} / \mathrm{m}^{2}$ i.v. on day 1 , capecitabine $1,000 \mathrm{mg} / \mathrm{m}^{2}$ orally twice daily from day 1 to day 11 (OXXEL); or oxaliplatin $85 \mathrm{mg} / \mathrm{m}^{2}$ i.v. on day 1 ; $6 \mathrm{~S}$-leucovorin $250 \mathrm{mg} / \mathrm{m}^{2}$ i.v. and fluorouracil $850 \mathrm{mg} / \mathrm{m}^{2}$ i.v. on day 2 (OXAFAFU).

Results Eleven complete and 42 partial responses were registered with OXXEL $(\mathrm{RR}=34 \%)$; six complete and 48 partial responses were obtained with OXAFAFU $(\mathrm{RR}=33 \%)$
\end{abstract}

P. Comella $(\bowtie)$

Department of Medical Oncology, National Tumour Institute,

Via M. Semmola, 80100 Naples, Italy

e-mail: pasqualecomella@ libero.it

B. Massidda

Chair of Medical Oncology, University Medical School,

Strada Statale 554, 09100 Cagliari, Italy

G. Filippelli

Depatment of Medical Oncology, City Hospital,

Via Promintesta, 87027 Paola, Italy

A. Farris

Chair of Medical Oncology, University Medical School,

Via S. Pietro 8, 07100 Sassari, Italy

D. Natale

Depatment of Medical Oncology, City Hospital,

via Nazionale Nord 99, 65017 Penne, Italy
$(P=0.999)$. Severe adverse events were less frequent (32 vs. $43 \%$ ) with OXXEL, which also reduced the occurrence of severe neutropenia (10 vs. $27 \%)$ and febrile neutropenia (6 vs. 13\%), but produced more gastric side effects ( 8 vs. $3 \%)$ and diarrhea (13 vs. $8 \%$ ). QoL did not differ across the two arms. Median PFS was 6.6 months in the OXXEL, and 6.5 months in the OXAFAFU arm (HR $=1.12, P=0.354)$. Median overall survival was 16.0 and 17.1 months $(\mathrm{HR}=1.01, P=0.883)$.

Conclusions OXXEL and OXAFAFU regimens were equally active in metastatic colorectal cancer. The choice should be based on patient preference and on pharmacoeconomic evaluations.

Keywords Capecitabine - Oxaliplatin - OXXEL regimen OXAFAFU regimen · Colorectal carcinoma .

Randomized trial

\section{G. Barberis}

Depatment of Medical Oncology, Villa Betania Hospital,

Via Argine, 80100 Naples, Italy

L. Maiorino

Depatment of Medical Oncology, San Gennaro Hospital,

Via San Gennaro dei Poveri 5, 80100 Naples, Italy

S. Palmeri

Chair of Medical Oncology, University Medical School,

Via del Vespro 127, 90100 Palermo, Italy

M. Cannone

Depatment of Medical Oncology, City Hospital,

Via Bovio, 70031 Canosa, Italy

G. Condemi

Depatment of Medical Oncology, City Hospital,

Via Zara, 89048 Siderno, Italy 


\section{Introduction}

Oxaliplatin as a single agent has demonstrated activity in both chemonaive and fluorouracil-pretreated colorectal cancer patients (Raymond et al. 1998; Becouarn et al. 1998; Díaz-Rubio et al. 1998; Machover et al. 1996). Moreover, in vitro studies on colon cancer cell lines have shown a synergism between oxaliplatin and fluorouracil, which was greater when oxaliplatin preceded a short fluorouracil exposure (Fischel et al. 1998).

In randomized clinical trials, oxaliplatin combined with fluorouracil and leucovorin produced a greater response rate and a longer time to progression than fluorouracil and leucovorin alone; however, median survival times were similar (Giacchetti et al. 2000; de Gramont et al. 2000). Thereafter, the FOLFOX4 regimen has been demonstrated to be significantly more effective in terms of response rate, time to progression, and overall survival than the irinotecan plus leucovorin/fluorouracil (IFL) i.v. bolus regimen (Goldberg et al. 2004). This observation was confirmed in a subsequent randomized trial comparing FOLFOX4 with a modified IFL regimen (Goldberg et al. 2006). Moreover, the SICOG trial 9801 compared oxaliplatin and irinotecan in combination with leucovorin-modulated fluorouracil i.v. bolus every 2 weeks. The OXAFAFU regimen produced a significantly greater response rate, and a significantly longer progression-free and overall survival than the IRIFAFU regimen, with a less pronounced toxicity (Comella et al. 2005).

More recently, the combination of oxaliplatin and capecitabine has been investigated in several multicentre phase II/III studies in metastatic colorectal cancer. The XELOX regimen (oxaliplatin $130 \mathrm{mg} / \mathrm{m}^{2}$ i.v. on day 1 , and capecitabine $2,000 \mathrm{mg} / \mathrm{m}^{2}$ orally days $1-14$, every 3 weeks) achieved a $55 \%$ response rate, and a 19.5 months median overall survival in a large multicentre international phase II trial (Cassidy et al. 2004). A similar combination, with oxaliplatin $120 \mathrm{mg} / \mathrm{m}^{2}$ on day 1 and capecitabine $2,500 \mathrm{mg} /$ $\mathrm{m}^{2}$ days $1-14$, obtained a $44 \%$ response rate, and a median survival time of 20 months; however, occurrence of grade $\geq 3$ diarrhea was seen in $28 \%$ of patients (Zeuli et al. 2003). Furthermore, a phase II randomized study assessed the addition of oxaliplatin $70 \mathrm{mg} / \mathrm{m}^{2}$ (CAPOX) or irinotecan $80 \mathrm{mg} / \mathrm{m}^{2}$ (CAPIRI) on days 1 and 8 to capecitabine $2,000 \mathrm{mg} / \mathrm{m}^{2}$ given for 2 weeks, reporting a $50.7 \%$ response rate with the CAPOX regimen (Grothey et al. 2003). In a Swiss phase II study, 26 pretreated and 43 chemonaive patients received oxaliplatin $130 \mathrm{mg} / \mathrm{m}^{2}$ and capecitabine $2,500 \mathrm{mg} / \mathrm{m}^{2}$ on day $1-14$ every 3 weeks. A response rate of $49 \%$, and a median overall survival time of 17.1 months were reported in previously untreated patients (Borner et al. 2002). The feasibility and activity of the XELOX regimen in elderly patients has also been investigated: a $37 \%$ response rate, with 8.5 months of median progression-free survival and 14.4 months of median survival, have been reported (Comella et al. 2005). These findings were subsequently confirmed by Feliu et al. (2006), who reported a $36 \%$ response rate, a median progression-free survival of 5.8 months, and an overall survival of 13.2 months.

Recently, we have assessed a different schedule of this combination, including oxaliplatin $100 \mathrm{mg} / \mathrm{m}^{2}$ on day 1 , and capecitabine $1,000 \mathrm{mg} / \mathrm{m}^{2}$ bid from day 1 (evening) to day 11 (morning), recycling every 2 weeks (OXXEL regimen). The rationale was to keep the same dose intensity for capecitabine as in the 3-weekly regimen, while slightly increasing the dose intensity of oxaliplatin. We achieved with this treatment a $45 \%$ response rate, and a median progression-free survival of 7.9 months; occurrence of severe side effects was negligible (Comella et al. 2005).

Based on these premises, we devised to conduct a randomized trial to compare the OXXEL and OXAFAFU regimens in metastatic colorectal cancer patients. Preliminary comparative data on the safety have already been reported (Sandomenico et al. 2006). Here we present the final results in terms of efficacy and quality of life of treated patients.

\section{Methods}

Patient selection

This trial was approved by the Independent Ethics Committee of the National Tumor Institute of Naples, and all participating patients provided a written informed consent. Eligible patients had histologically proven diagnosis of advanced adenocarcinoma of the colon or rectum, age $\geq 18$ years, life expectancy $>3$ months, and ECOG performance status $\leq 2$. Additional inclusion criteria were: adjuvant chemotherapy completed at least 6 months before, presence of a bidimensionally measurable lesion, neutrophil count $\geq 2 \times 10^{6} / \mathrm{L}$, platelet count $\geq 100 \times 10^{6} / \mathrm{L}$, hemoglobin level $\geq 100 \mathrm{~g} / \mathrm{L}$, serum bilirubin $\leq 1.25$ times the upper normal limit (UNL), serum alanine aminotransferase and aspartate aminotransferase $\leq 2.5 \times \mathrm{UNL}$ in absence of liver metastasis, or $\leq 5 \times \mathrm{NL}$ in presence of liver metastasis; normal renal function.

\section{Patients evaluation}

Biochemistry profile, blood cell count with white blood cell (WBC) count and differential, and carcinoembryonic antigen (CEA) serum level assessment, were performed at baseline. Target lesions were measured by computed tomography (CT) or magnetic resonance imaging (MRI) scans not more than 4 weeks before initial therapy. During 
treatment, WBC count with differential was performed weekly. Biochemistry, symptoms, body weight, and nonhematological toxicity were checked before each cycle. Toxicity was scored according to WHO criteria (Miller et al. 1981), while neuropathy was defined according to the Lévi scale (Lévi et al. 1992), and the worst toxicity suffered by each patient during the whole treatment was recorded.

Patients were required to fulfill the European Organization for Research and Treatment of Cancer Quality of Life questionnaire (EORTC QLQ-C30 version 3.0) before randomization, and every 8 weeks during treatment. Changes $>10$ points of the baseline scores were considered clinically meaningful (Osoba et al. 1998).

CT or MRI scan was repeated after every 4 cycles, and at the end of treatment. Response was defined according to WHO criteria (Miller et al. 1981), and reassessed 8 weeks after the date of their first documentation; only confirmed responses were computed in the activity analysis.

\section{Treatment}

Patients, after stratification according centre, performance status, and previous exposure to adjuvant chemotherapy, were randomly allocated to receive: oxaliplatin $100 \mathrm{mg} / \mathrm{m}^{2}$ i.v. (2 h) on day 1 ; capecitabine $1,000 \mathrm{mg} / \mathrm{m}^{2}$ orally twice daily (12-h apart) from day 1 (evening) to day 11 (morning) (OXXEL regimen); or oxaliplatin $85 \mathrm{mg} / \mathrm{m}^{2}$ i.v. ( $2 \mathrm{~h}$ ) on day 1; 6S-leucovorin $250 \mathrm{mg} / \mathrm{m}^{2}$ i.v. ( $2 \mathrm{~h}$ ), followed by fluorouracil $850 \mathrm{mg} / \mathrm{m}^{2}$ i.v. bolus on day 2 (OXAFAFU regimen). In both arms, cycles were repeated every 2 weeks, until progression, unacceptable toxicity or patient refusal, or for a maximum of 12 cycles.

After discontinuation of first-line treatment, patients were followed every 2 months to assess the disease status and survival. Further treatment was not planned, and it was left to the single investigator choice.

\section{Statistical considerations and analysis}

Primary end-point of this study was to compare the activity of the two regimens. Since a great difference was unlikely on the ground of our previous trials, we planned to have an $80 \%$ power to demonstrate, with an alpha error $=0.05$, a $15 \%$ minimum difference in response rate between the two arms. The planned accrual was 150 patients per arm. This sample size also allowed to make a comparison of progression-free survival. Indeed, 257 events had a $90 \%$ power to demonstrate, with an alpha error $=0.05$, a $33 \%$ reduction of the hazard of progression. The predefined target population had also an $80 \%$ power to demonstrate (with alpha error $=0.05$ ) a $20 \%$ difference in the proportion of patients showing a QoL preservation (i.e., $a<10$-point decrease from the baseline score) after 8 weeks from initial therapy.
Frequencies were calculated with their $95 \%$ confidence limits (CL), and compared with the chi-squared or Fisher's exact test. A logistic regression analysis was performed to ascertain the independent effect on response of some baseline patient characteristics: sex, primary site, previous surgery, previous adjuvant chemotherapy, previous loss $>5 \%$ of body weight, performance status, abnormal CEA serum level, presence of synchronous metastases, presence of liver metastases, or metastases confined to liver were included as dichotomous variables, while age, tumor grading, serum alkaline phosphatase concentration, and number of disease sites were included as continuous variables. Time-to event probabilities were estimated with the Kaplan and Meier method (Kaplan and Meier 1958), and compared with the two-sided log-rank test (Mantel 1966). Baseline demographic and clinical characteristics were included in a Cox multivariate analysis (Cox 1972) to assess their independent effect on failure-free, progression-free, and overall survival.

\section{Results}

From May 2004 to April 2007, 344 patients were registered into this study. However, 12 patients did not met all the inclusion criteria, leaving 322 eligible patients, who were randomized to the OXXEL (158 patients) or OXAFAFU (164 patients) arm. Baseline characteristics were usually well balanced between the two arms of treatment. However, there were more males and more patients with liver only metastases in the OXXEL arm. Conversely, more patients in the OXAFAFU arm had an elevated CEA basal value (Table 1).

Delivered treatment and toxicity

A total of 1,251 cycles of OXXEL, and 1,282 cycles of OXAFAFU were delivered, with a median number of eight (range 1-12) cycles/patient in both arms. Comparable proportions of patients in the two arms received 4,8 or 12 cycles (Table 2). Median duration of treatment was 17 (range 1-36) weeks in either arm.

Median cumulative oxaliplatin dose was significantly greater $(P=0.001)$ for patients treated with OXXEL $\left(739 \mathrm{mg} / \mathrm{m}^{2}\right.$, range $75-1,232 \mathrm{mg} / \mathrm{m}^{2}$ ) than with OXAFAFU $\left(659 \mathrm{mg} / \mathrm{m}^{2}\right.$, range $\left.63-1,069 \mathrm{mg} / \mathrm{m}^{2}\right)$. Similarly, median dose intensity of oxaliplatin was higher for the former (43 $\mathrm{mg} / \mathrm{m}^{2}$ per week, range $14-81 \mathrm{mg} / \mathrm{m}^{2}$ per week) than for the latter $\left(34 \mathrm{mg} / \mathrm{m}^{2}\right.$ per week, range, $13-78 \mathrm{mg} /$ $\mathrm{m}^{2}$ per week) $(P=0.001)$. Median relative dose intensities of this drug were similar in the two arms (84 vs. $80 \%)$. 
Table 1 Demographic and clinical characteristic according to arms of treatment

\begin{tabular}{|c|c|c|c|c|c|c|c|}
\hline \multirow[t]{2}{*}{ Arm characteristics } & \multicolumn{2}{|c|}{ OXAFAFU } & \multirow{2}{*}{$\begin{array}{l}\text { Fisher's } \\
\text { test }\end{array}$} & \multicolumn{2}{|c|}{ OXXEL } & \multicolumn{2}{|c|}{ Total } \\
\hline & No. & $\%$ & & No. & $\%$ & No. & $\%$ \\
\hline Eligible patients & 164 & 100 & & 158 & 100 & 322 & 100 \\
\hline Males & 89 & 54 & 0.023 & 104 & 66 & 193 & 58 \\
\hline Females & 75 & 46 & & 54 & 34 & 129 & 42 \\
\hline Median age (range) & \multicolumn{3}{|c|}{$65(37-79)$} & \multicolumn{2}{|c|}{$64(39-84)$} & \multicolumn{2}{|c|}{$63(37-84)$} \\
\hline Aged $\geq 70$ years & 65 & 40 & & 51 & 32 & 116 & 36 \\
\hline \multicolumn{8}{|l|}{ Primary tumor } \\
\hline Colon & 115 & 76 & & 114 & 72 & 229 & 71 \\
\hline Rectum & 49 & 24 & & 44 & 28 & 93 & 29 \\
\hline \multicolumn{8}{|l|}{ Grading } \\
\hline Well differentiated & 14 & 9 & & 10 & 6 & 24 & 7 \\
\hline Moderately differentiated & 92 & 56 & & 103 & 65 & 195 & 61 \\
\hline Poorly differentiated & 31 & 19 & & 29 & 18 & 60 & 19 \\
\hline Unknown & 27 & 16 & & 16 & 10 & 43 & 13 \\
\hline Previous surgery & 125 & 76 & & 114 & 72 & 239 & 74 \\
\hline Previous adjuvant chemotherapy & 41 & 25 & & 39 & 25 & 80 & 25 \\
\hline \multicolumn{8}{|l|}{ ECOG Performance Status } \\
\hline 0 & 99 & 60 & & 96 & 61 & 195 & 61 \\
\hline 1 & 59 & 36 & & 57 & 36 & 116 & 36 \\
\hline 2 & 6 & 4 & & 5 & 3 & 87 & 3 \\
\hline \multicolumn{8}{|l|}{ No. disease sites } \\
\hline 1 & 74 & 45 & & 79 & 50 & 153 & 48 \\
\hline 2 & 55 & 33 & & 45 & 29 & 100 & 31 \\
\hline $3+$ & 35 & 21 & & 34 & 21 & 69 & 21 \\
\hline Liver positive & 123 & 75 & & 131 & 83 & 254 & 79 \\
\hline Liver only & 48 & 29 & 0.023 & 64 & 41 & 112 & 35 \\
\hline Synchronous metastasis & 96 & 59 & & 91 & 58 & 124 & 58 \\
\hline Weigh loss $\geq 5 \%$ & 40 & 24 & & 44 & 28 & 84 & 26 \\
\hline Alkaline phosphatase $>$ UNL & 59 & 36 & & 53 & 34 & 112 & 35 \\
\hline \multicolumn{8}{|l|}{ CEA value } \\
\hline$>5 \mathrm{ng} / \mathrm{mL}$ & 143 & 87 & 0.007 & 120 & 76 & 263 & 82 \\
\hline$>100 \mathrm{U} / \mathrm{mL}$ & 40 & 24 & & 33 & 21 & 73 & 23 \\
\hline
\end{tabular}

In the OXXEL arm, median dose intensity for capecitabine was 8,046 (range $5,450-12,000$ ) $\mathrm{mg} / \mathrm{m}^{2}$ per week ( $80 \%$ of the planned one); in the OXAFAFU arm, median dose intensity of fluorouracil was 308 (range 153-406) mg/ $\mathrm{m}^{2}$ per week ( $72 \%$ of the intended one).

Occurrence of severe neutropenia (10 vs. 27\%, $P<0.001$ ) and febrile neutropenia (6 vs. $13 \%, P=0.043$ ) was significantly lower with the OXXEL treatment, while frequencies of grade $\geq 3$ thrombocytopenia (4 vs. $3 \%$ ) and anemia ( 3 vs. $1 \%$ ) were similar. Severe diarrhea affected more patients treated with OXXEL (13 vs. $8 \%$ ), but this difference was not significant. However, gastric intolerance was more common with the oral assumption of capecitabine ( 8 vs. $3 \%, P=0.028$ ). Other non-hematological side effects were registered in few patients, and were comparable in both arms (Table 3). Despite the greater amount of oxaliplatin delivered with OXXEL, this treatment did not produce more severe neuropathy than OXAFAFU regimen (10 vs. 7\%). On the whole, treatment-related severe adverse events affected significantly less patients in OXXEL than in OXAFAFU arm (32 vs. 43\%, $P=0.026$ ). Early deaths (within 60 days from initial therapy) were registered in similar proportions in both arms (3 vs. 4\%). In OXXEL arm, a toxic death was caused by severe diarrhea and dehydration in two elderly patients. These patients had previously received several cycles of chemotherapy without experiencing severe toxicity, and had a normal renal function.

Activity

Eleven complete and 42 partial responses were registered in the OXXEL arm, for a response rate of $34 \%$; six complete and 
Table 2 Treatment disposition in the two arms of treatment

\begin{tabular}{|c|c|c|c|c|c|c|c|}
\hline \multirow{2}{*}{$\begin{array}{l}\text { Arm } \\
\text { characteristics }\end{array}$} & \multicolumn{2}{|c|}{ OXAFAFU } & \multirow{2}{*}{$\begin{array}{l}\text { Fisher's } \\
\text { test }\end{array}$} & \multicolumn{2}{|c|}{ OXXEL } & \multicolumn{2}{|l|}{ Total } \\
\hline & No. & $\%$ & & No. & $\%$ & No. & $\%$ \\
\hline Eligible patients & 164 & 100 & & 158 & 100 & 322 & 100 \\
\hline Total number of cycles & 1,272 & & & 1,243 & & 2,515 & \\
\hline Median cycles/patient (range) & 8 & $1-12$ & & 8 & $1-12$ & 8 & $1-12$ \\
\hline \multicolumn{8}{|l|}{ Patients treated with: } \\
\hline$\geq 4$ cycles & 146 & 89 & & 141 & 89 & 287 & 89 \\
\hline$\geq 8$ cycles & 97 & 59 & & 96 & 61 & 193 & 60 \\
\hline$\geq 12$ cycles & 46 & 28 & & 45 & 28 & 91 & 28 \\
\hline Patients still on therapy & 7 & 4 & & 7 & 4 & 14 & 4 \\
\hline \multicolumn{8}{|l|}{ Patients off treatment for: } \\
\hline Protocol & 110 & 67 & & 101 & 64 & 210 & 65 \\
\hline Refusal & 13 & 8 & & 16 & 10 & 29 & 9 \\
\hline Toxicity & 10 & 6 & 0.015 & 21 & 13 & 32 & 10 \\
\hline Disease complications & 14 & 8 & & 5 & 3 & 19 & 6 \\
\hline Physician's decision & 10 & 6 & & 8 & 5 & 18 & 5 \\
\hline
\end{tabular}

Table 3 Frequencies of main side effects according to arms of treatment

ns not significant

\begin{tabular}{|c|c|c|c|c|c|c|}
\hline \multirow{2}{*}{$\begin{array}{l}\text { Arm } \\
\text { WHO toxicity (\%) }\end{array}$} & \multicolumn{2}{|c|}{ OXAFAFU } & \multicolumn{2}{|l|}{ OXXEL } & \multicolumn{2}{|l|}{ Fisher's test } \\
\hline & All grades & Grade $\geq 3$ & All grades & Grade $\geq 3$ & All grades & Grade $\geq 3$ \\
\hline Neutropenia & 49 & 27 & 15 & 10 & 0.001 & 0.001 \\
\hline Febrile neutropenia & - & 13 & - & 6 & - & 0.043 \\
\hline Anemia & 30 & 1 & 23 & 3 & ns & ns \\
\hline Thrombocytopenia & 21 & 3 & 24 & 4 & ns & ns \\
\hline Diarrhea & 43 & 8 & 36 & 13 & ns & ns \\
\hline Neuropathy & 43 & 7 & 48 & 10 & ns & ns \\
\hline Gastric symptoms & 41 & 3 & 50 & 8 & ns & 0.028 \\
\hline Stomatitis & 18 & 2 & 15 & 2 & ns & ns \\
\hline Liver toxicity & 15 & 1 & 22 & 0 & ns & ns \\
\hline Hair loss & 14 & 0 & 7 & 0.6 & ns & ns \\
\hline Hand \& foot syndrome & 10 & 1 & 15 & 4 & ns & $\mathrm{ns}$ \\
\hline Renal toxicity & 4 & 0.6 & 8 & 2 & ns & ns \\
\hline Allergic reactions & 4 & 3 & 3 & 0.6 & ns & ns \\
\hline Fatigue & 4 & 1 & 5 & 1 & ns & ns \\
\hline
\end{tabular}

48 partial responses were registered in the OXAFAFU arm, for a response rate of $33 \%$ (odds ratio $=1.03,95 \%$ CL, 0.63 $1.68, P=0.999$ ). An overall disease control (response or stabilization) was achieved in 68 and $70 \%$ patients, respectively.

Regardless of received treatment, response rate was slightly higher in patients with synchronous metastases (37 vs. $27 \%$ ), and in younger ( $\leq 60$-year-old) patients (40 vs. $30 \%$ ). At the multivariate analysis, only age of patients (as continuous variable) adversely affected the probability of response $(P<0.001)$.

Median failure-free survival was 4.9 (95\% CL, $4.2-$ 5.6) months for patients treated with OXXEL, and 4.7
(95\% CL, 4.0-5.4) months for patients treated with OXAFAFU (hazard ratio $[\mathrm{HR}]=0.92,95 \% \mathrm{CL}, 0.73-1.17$; $P=0.555)$. At Cox analysis, only the number of disease sites was significantly associated with a shorter failure-free survival $(P=0.049)$.

Median progression-free survival was $6.6(95 \% \mathrm{CL}$, 6.0-7.0) for patients treated with OXXEL, and 6.5 (95\% CL, 5.4-7.6) months for those treated with OXAFAFU $(\mathrm{HR}=1.12,95 \% \mathrm{CL}, 0.88-1.45, P=0.354)$ (Fig. 1). Number of disease sites $(P=0.001)$, followed by an elevated basal CEA value $(P=0.036)$ were negative factors for progression-free survival. 


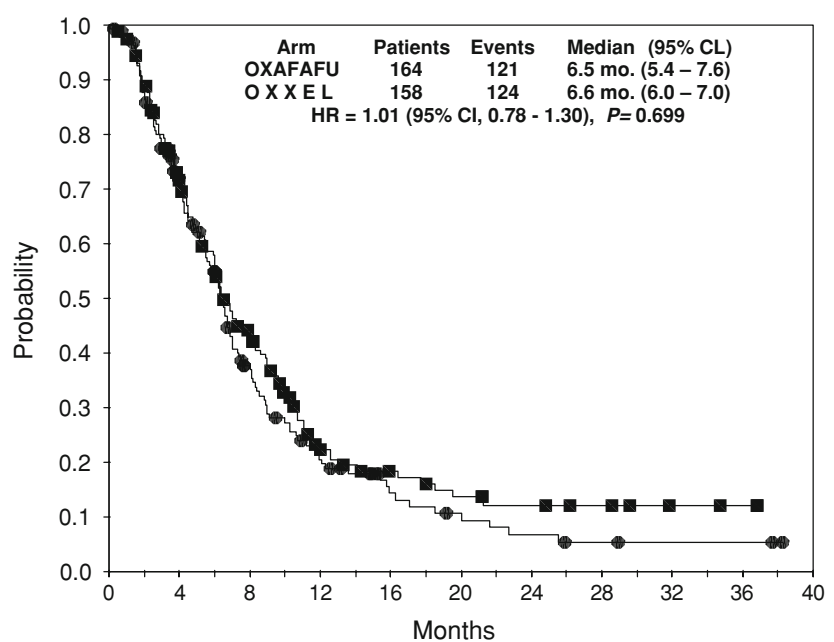

Fig. 1 Estimated progression-free survival curves according to arms of treatment (OXAFAFU squares, OXXEL circles)

Quality of life evaluation

Three-hundred and twelve (97\%) eligible patients (OXXEL arm, 151 patients; OXAFAFU arm, 161 patients) filled in the baseline questionnaire. After 8 weeks, the questionnaire was available for 225 of 287 (78\%) patients on therapy. After 16 weeks, 193 patients were on therapy, and questionnaires were available for $156(81 \%)$ patients. Finally, after 24 weeks, the available questionnaires were $72(79 \%)$ of 91 patients still on therapy.

Baseline single item and global health status/quality of life scores did not significantly differ between the two arms. Excluding constipation $(P=0.001)$ and financial item score $(P=0.004)$, no other significant differences in the change of single scores were observed between the two arms during the whole treatment (Table 4).

At the predetermined time-point for the comparison, a preservation of the quality of life was reported in $47 \%$ of patients in either arm. After 16 weeks, a higher proportion of patients in the OXXEL than in OXAFAFU arm showed a deterioration of the global health status/quality of life score; the same trend was also observed after 24 weeks. However, these differences were not statistically significant (Table 5).

\section{Overall survival}

As of October 2007, after a median follow-up of 24 (range 6-42) months, $162(50 \%)$ patients have died (78 patients in the OXXEL, and 84 patients in the OXAFAFU arm). Median overall survival was 16.0 (95\% CL, 11.2 20.2) months, and 17.1 (95\% CL, 13.8-20.4) months, respectively $(\mathrm{HR}=1.01,95 \% \mathrm{CL}, 0.74-1.38, P=0.883$ ). One-, 2- and 3-year probabilities of survival were 59, 36 and $31 \%$ for the OXXEL arm, and 63,35 and $26 \%$ for the OXAFAFU arm (Fig. 2).

Table 4 Quality of life of patients according to arms of treatment (values are means and standard errors)

\begin{tabular}{|c|c|c|c|c|c|c|c|c|}
\hline & \multicolumn{2}{|c|}{ Baseline assessment } & \multicolumn{2}{|c|}{ After 8 weeks } & \multicolumn{2}{|c|}{ After 16 weeks } & \multicolumn{2}{|c|}{ After 24 weeks } \\
\hline & OXXEL & OXAFAFU & OXXEL & OXAFAFU & OXXEL & OXAFAFU & OXXEL & OXAFAFU \\
\hline & $151^{\mathrm{a}}$ & $161^{\mathrm{a}}$ & $107^{\mathrm{a}}$ & $118^{\mathrm{a}}$ & $83^{\mathrm{a}}$ & $73^{\mathrm{a}}$ & $33^{\mathrm{a}}$ & $39^{\mathrm{a}}$ \\
\hline \multicolumn{9}{|l|}{ Items } \\
\hline Physical & $81(1.4)$ & $80(1.5)$ & $79(2.1)$ & $75(1.8)$ & $80(2.1)$ & $74(2.6)$ & $83(4.6)$ & $74(2.6)$ \\
\hline Role & $76(2.4)$ & $75(2.1)$ & $76(2.7)$ & $68(3.4)$ & $79(2.5)$ & $76(3.6)$ & $77(5.7)$ & $85(3.6)$ \\
\hline Emotional & $72(1.7)$ & $68(1.7)$ & $70(2.2)$ & $71(2.1)$ & $74(2.1)$ & $72(2.9)$ & $72(3.8)$ & $75(3.7)$ \\
\hline Cognitive & 87 (1.7) & $85(1.5)$ & $82(2.7)$ & $83(1.8)$ & $85(2.3)$ & $82(3.0)$ & $86(4.2)$ & $77(9.0)$ \\
\hline Social & $82(1.8)$ & $80(2.1)$ & $78(2.5)$ & $77(2.1)$ & 77 (2.9) & $79(3.1)$ & $78(5.2)$ & $87(3.1)$ \\
\hline Fatigue & $28(1.9)$ & $30(1.9)$ & $31(2.5)$ & $37(2.4)$ & $30(2.2)$ & $38(3.9)$ & $34(5.1)$ & $28(3.6)$ \\
\hline Nausea/Vomiting & $5(0.9)$ & $6(1.3)$ & $15(2.3)$ & $13(1.6)$ & $12(1.7)$ & $12(2.4)$ & $10(3.8)$ & $4(1.5)$ \\
\hline Pain & $18(1.9)$ & $13(1.5)$ & $23(4.1)$ & $21(2.2)$ & $15(2.5)$ & $16(2.9)$ & $23(4.8)$ & $13(2.9)$ \\
\hline Dyspnoea & $9(1.4)$ & $13(1.6)$ & $12(2.3)$ & $14(1.6)$ & $11(2.2)$ & $12(2.5)$ & $19(4.7)$ & $9(2.9)$ \\
\hline Insomnia & $25(2.3)$ & $31(2.4)$ & $26(2.9)$ & $24(2.4)$ & $20(2.8)$ & $23(3.3)$ & $29(5.5)$ & $16(3.7)$ \\
\hline Appetite loss & $16(2.1)$ & $18(1.9)$ & $23(2.1)$ & $21(1.9)$ & $19(2.9)$ & $22(3.4)$ & $16(4.5)$ & $11(3.1)$ \\
\hline Constipation & $20(2.4)$ & $20(2.1)$ & $16(2.6)$ & $22(2.7)$ & $13(2.6)$ & $27(3.5)$ & $10(3.2)$ & $15(3.4)$ \\
\hline Diarrhoea & $9(1.5)$ & $10(1.5)$ & $16(2.4)$ & $20(2.5)$ & $14(2.2)$ & $15(2.8)$ & $10(3.6)$ & $11(3.3)$ \\
\hline Financial difficulties & $17(2.2)$ & $20(2.1)$ & $19(2.8)$ & $18(1.8)$ & $19(3.0)$ & $21(3.9)$ & $25(5.5)$ & $19(2.9)$ \\
\hline General health status & $66(1.8)$ & $65(1.7)$ & $65(2.2)$ & $65(1.8)$ & $70(2.2)$ & $67(2.5)$ & $67(5.1)$ & $69(2.9)$ \\
\hline
\end{tabular}

\footnotetext{
${ }^{a}$ Number of patients assessed
} 
Table 5 Patients showing a significant change of the quality of life score during treatment: improved means a $\geq 10$ points increment of the baseline score; deteriorated means a $\geq 10$-point decrease of the baseline score

\begin{tabular}{|c|c|c|c|c|c|c|c|c|c|}
\hline \multirow[t]{2}{*}{ Arm } & \multicolumn{3}{|c|}{ After 8 weeks } & \multicolumn{3}{|c|}{ After 16 weeks } & \multicolumn{3}{|c|}{ After 24 weeks } \\
\hline & Improved & Stable & Deteriorated & Improved & Stable & Deteriorated & Improved & Stable & Deteriorated \\
\hline OXXEL & $24(23 \%)$ & $50(47 \%)$ & $30(30 \%)$ & $30(37 \%)$ & $29(35 \%)$ & $23(28 \%)$ & $5(17 \%)$ & $2(7 \%)$ & $47(76 \%)$ \\
\hline OXAFAFU & $25(22 \%)$ & $56(47 \%)$ & $37(31 \%)$ & $17(24 \%)$ & $40(57 \%)$ & $13(19 \%)$ & $1(3 \%)$ & $7(18 \%)$ & $30(79 \%)$ \\
\hline
\end{tabular}

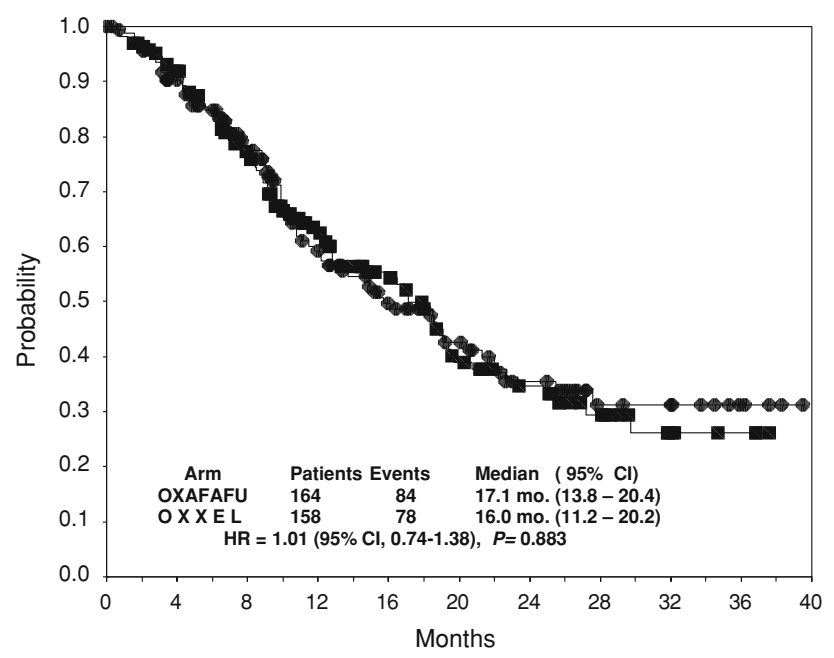

Fig. 2 Estimated overall survival curves according to arms of treatment (OXAFAFU squares, OXXEL circles)

\section{Discussion}

The findings of this study clearly showed that there was no difference in activity between OXAFAFU and OXXEL regimens. Indeed, response rates (33 vs. $34 \%$ ), and median progression-free survivals ( 6.5 vs. 6.6 months) were comparable, and the $95 \%$ CLs of these results were quite overlapping. However, we should remember that the trial was not powered to demonstrate the non-inferiority of the OXXEL in comparison with the OXAFAFU regimen. Therefore, we cannot exclude that patients treated with the former regimen may have a shorter time to progression than patients treated with the latter.

Moreover, we have to admit that response rate and progression-free survival of patients treated with oxaliplatin plus a fluoropyrimidine in the present trial appear slightly worse than those reported with oxaliplatin and fluorouracil/ leucovorin in our previous study (Comella et al. 2005). However, we would underline that the present trial, patients aged $\geq 70$ years represented $36 \%$ of the whole series, as opposed to $20 \%$ of the previous trial, and we have shown that age was an independent risk factor adversely affecting the response rate in the present study. Moreover, nearly twice more patients $(21 \%)$ in the present series, as opposed to $11 \%$ in the previous trial, had $\geq 3$ disease sites, and the number of involved sites was independently related with a shorter failure-free and progression-free survival in the present study.

As for side effects, we may state that OXXEL was better tolerated than the OXAFAFU regimen. Although severe and febrile neutropenia were infrequent with both regimens, these side effects were significantly reduced with the OXXEL treatment. This safety gain was not counterbalanced by non-hematologic side effects. Indeed, only gastric intolerance was slightly more pronounced with the substitution of oral capecitabine for i.v. fluorouracil/leucovorin.

Regarding quality of life, the diarrhea score derived by the questionnaires was similar in the two arms, while the constipation score showed a slight but significant improvement in patients treated with OXXEL. Of course, we have to remember the limitation of these comparisons, which may have been biased by the mild attrition of patients answering the questionnaire.

To put our results in perspective, we have to mention some randomized trials that also compared the combination of oxaliplatin with either capecitabine or i.v. fluorouracil in metastatic colorectal cancer patients.

In a phase II randomized trial, 118 patients received either the XELOX regimen or oxaliplatin $130 \mathrm{mg} / \mathrm{m}^{2}$ every 3 weeks combined with fluorouracil $250 \mathrm{mg} / \mathrm{m}^{2}$ daily as protracted i.v. infusion for 3 weeks. The activity was similar (response rate, 43.5 vs. $48.2 \%$; median progression-free survival, 9 vs. 7 months). However, the XELOX regimen caused less severe diarrhea ( 8 vs. $13 \%$ ), and stomatitis (13 vs. 29\%) (Martoni et al. 2006).

In the TREE-1 phase II study, 157 patients randomly received: mFOLFOX, bFOL, or XELOX regimen. Response rates were 43,22 , and $35 \%$; median progressionfree survival were 8.7, 6.9, and 5.9 months, and median overall survival were $19.2,17.9$, and 17.2 months, respectively. The XELOX regimen produced more severe dehydration $(27 \%)$ as opposed to mFOLFOX or bFOL regimens, while severe diarrhea had a similar occurrence with all these regimens. The safety advantage of the XELOX regimen was limited to neutropenia, which was much lower (15\%) than that reported with mFOLFOX (53\%) (Hochster et al. 2006).

A German phase III trial compared in 474 patients the CAPOX regimen with the FUFOX regimen. The former 
produced slightly worse response rate (48 vs. 54\%, $P=0.7)$, median progression-free survival (7.1 vs. 8.0 months. $\mathrm{HR}=1.17,95 \% \mathrm{CL}, 0.96-1.43, P=0.117)$, and overall survival (16.8 vs. 18.8 months, $\mathrm{HR}=1.12,95 \%$ CL, 0.92-1.38, $P=0.260$ ) than the infusional regimen; no safety advantage has been reported for CAPOX, which produced a significantly greater occurrence of grade 2-3 handfoot syndrome (Porschen et al. 2007).

A Spanish phase IIII trial compared the XELOX regimen with a regimen including weekly infusional fluorouracil plus biweekly oxaliplatin in 348 patients. Although patients treated with XELOX had a lower response rate (37 vs. $46 \%, P=0.539)$, and a shorter median time to progression (8.9 vs. 9.5 months, $\mathrm{HR}=1.18$, 95\% CL, 0.9-1.5, $P=0.153)$ and overall survival (18.1 vs. 20.8 months, $\mathrm{HR}=1.22,95 \% \mathrm{CL}, 0.9-1.6, P=0.145)$, these differences were not significant. In this study, less patients treated with XELOX suffered from severe diarrhea (14\% vs. 24\%). However, XELOX was associated with more hand-foot syndrome (14 vs. 5\%) (Díaz-Rubio et al. 2007).

The NO16966 phase III trial compared the XELOX and FOLFOX4 regimens, before and after the introduction in clinical practice of bevacizumab. A total of 634 patients were treated without bevacizumab: the response rate was 37 vs. $39 \%$, and the median progression-free survival was 7.3 vs. 7.7 months, respectively ( $\mathrm{HR}=0.96,97.5 \% \mathrm{CL}, 0.80-1.16)$. Given the confidence limits of these results, the non-inferiority of the XELOX regimen could be accepted. The XELOX reduced the risk of neutropenia (7vs.43\%), but produced more diarrhea (20 vs. $11 \%$ ) and skin toxicity (6 vs. $1 \%$ ) than the FOLFOX4 regimen (Cassidy et al. 2007). Subsequently, the addition of bevacizumab or placebo to either FOLFOX4 or XELOX did not increase the overall response rate, which indeed was $47 \%$ with bevacizumab and $49 \%$ with placebo. Bevacizumab significantly prolonged the progression-free survival (from 8.0 to 9.4 months, $\mathrm{HR}=0.83, P=0.0023$ ), but this advantage was significant only for patients treated with XELOX. Excluding the occurrence of hypertension (3.7 vs. $1.2 \%$ ), severe toxicity was not significantly worsened by the addition of bevacizumab (Salts et al. 2007).

A French phase III trial randomly compared XELOX and FOLFOX6 regimens in 306 metastatic patients. The non-inferiority of the XELOX regimen was proven, because the response rate was 39 vs. $46 \%$, and the $95 \%$ upper limit of this difference was below the non-inferiority margin; median progression-free survival was 8.8 vs. 9.3 months $(\mathrm{HR}=1.0,95 \% \mathrm{CL}, 0.82-1.22)$, and median overall survival was 19.9 vs. 20.5 months $(\mathrm{HR}=1.02,95 \%$ CL, 0.81-1.30). XELOX significantly reduced the occurrence of neutropenia ( 7 vs. $43 \%$ ), febrile neutropenia (0 vs. $6 \%$ ), and neuropathy (25 vs. 11\%) (Ducreux et al. 2007).

In conclusion, the comparable activity of regimens including oxaliplatin with either oral capecitabine or bolus, short or protracted i.v. infusion of fluorouracil (with or without leucovorin) is consistently supported by all these randomized trials, although it has been formally proven in only two of them (Cassidy et al. 2007; Ducreux et al. 2007). However, a safety advantage for the oral instead of intravenous delivery of fluoropyrimidine has not been clearly established.

In our study, the assessment of quality of life of patients during treatment did not show relevant differences. Other considerations, like patient's preference for an oral therapy, and pharmacoeconomic analyses, could play a role in the choice for the combination regimen. Some investigators have underlined the patient preference for an oral and/or home therapy, provided that it is equally effective to an intravenous regimen (Liu et al. 1997; Twelves et al. 2006); others reported a grater preference for an i.v. regimen, which produced lower acute toxicity (Pfeiffer et al. 2006). Moreover, some studies have shown that capecitabine is associated with reduced costs compared with i.v. fluorouracil/leucovorin in both the adjuvant and palliative setting (Cassidy et al. 2006; Ward et al. 2006), and that the majority of cost savings were due to the reduced administration costs. The additional cost of combining oxaliplatin with capecitabine instead of fluorouracil/leucovorin (Mayer 2007) could be counterbalanced by the lower incidence of some lifethreatening adverse events, translating into reduced costs for hospitalization. Moreover, future researches should further elucidate the optimal role of bevacizumab in the management of metastatic colorectal cancer patients, in order to achieve the maximum level of clinical benefit.

Acknowledgments The following investigators of the Southern Italy Cooperative Oncology Group are acknowledged as contributors to this trial: Rossana Casaretti MD, Claudia Sandomenico MD, Department of Medical Oncology, National Tumour Institute, Naples; Silvana Leo MD, Medical Oncology, City Hospital, Lecce; Salvatore Tafuto MD, Medical Oncology, City Hospital, Pozzuoli; Ettore Greco MD, Medical Oncology, City Hospital, Lametia Terme; Mario Roselli PhD, Chair of Medical Oncology, Tor Vergata University Medical School, Rome; Valerio Milia MD, Medical Oncology, City Hospital, Macomer; Antonio Gambardella PhD, Chair of Geriatrics, Second University Medical School, Naples; Sergio Mancarella MD, Medical Oncology, City Hospital, Galatina; Giancarlo Di Pinto MD, Medical Oncology, City Hospital, Castrovillari; Lucio De Luca MD, Medical Oncology, Pellegrini Hospital, Naples; Giacomo Vessia MD, Medical Oncology, City Hospital, Altamura, Italy. This work was partially supported by Italian Ministry of Health (FSN 2004). We thank Luca Franco and Marina Licenziato for their invaluable contribution in the data management.

Conflict of interest statement None to declare.

\section{References}

Becouarn Y, Ychou M, Ducreux M et al (1998) Phase II trial of oxaliplatin as first-line chemotherapy in metastatic colorectal cancer patients. J Clin Oncol 16:2739-2744 
Borner MM, Dietrich D, Stupp R et al (2002) Phase II study of capecitabine and oxaliplatin in first- and second-line treatment of advanced or metastatic colorectal cancer. J Clin Oncol 20:17591766

Cassidy J, Tabernero J, Twelves C et al (2004) XELOX (capecitabine plus oxaliplatin): active first-line therapy for patients with metastatic colorectal cancer. J Clin Oncol 22:2084-2091

Cassidy J, Douillard J-Y, Twelves C et al (2006) Pharmacoeconomic analysis of adjuvant oral capecitabine vs intravenous 5-FU/LV in Dukes' C colon cancer: the X-ACT trial. Br J Cancer 94:11221129

Cassidy J, Clarke S, Diaz-Rubio E et al. (2007) XELOX vs FOLFOX4: efficacy results from XELOX-1/NO16966, a randomised phase III trial in first-line metastatic colorectal cancer (MCRC). Proc Am Soc Clin Oncol Gastrointestinal Cancer Symposium (abstr 270)

Comella P, Massidda B, Filippelli G et al (2005a) Oxaliplatin plus high-dose folinic acid and 5-fluorouracil i.v. bolus (OXAFAFU) versus irinotecan plus high-dose folinic acid and 5-fluorouracil i.v. bolus (IRIFAFU) in patients with metastatic colorectal carcinoma: a Southern Italy Cooperative Oncology Group phase III trial. Ann Oncol 16:878-886

Comella P, Natale D, Farris A et al (2005b) Capecitabine plus oxaliplatin for the first-line treatment of elderly patients with metastatic colorectal carcinoma. Cancer 104:282-289

Comella P, Massidda B, Palmeri S et al (2005c) Biweekly oxaliplatin combined with oral capecitabine (OXXEL regimen) as first-line treatment of metastatic colorectal cancer patients. A Southern Italy Cooperative oncology group phase II study. Cancer Chemother Pharmacol 56:481-486

Cox DR (1972) Regression models and life tables. J R Stat Soc 34:187-202

Díaz-Rubio E, Sastre J, Zaniboni A et al (1998) Oxaliplatin as single agent in previously untreated colorectal carcinoma patients: a phase II multicentric study. Ann Oncol 9:105-108

Díaz-Rubio E, Tabernero J, Gómez-Espaňa J et al (2007) Phase III study of capecitabine plus oxaliplatin compared with continuousinfusion fluorouracil plus oxaliplatin as first-line therapy in metastatic colorectal cancer: final report of the Spanish cooperative group for the treatment of digestive tumors trial. J Clin Oncol 25:4224-4230

Ducreux M, Bennouna J, Hebbar M et al. (2007) Efficacy and safety findings from a randomised phase III study of capecitabine $(\mathrm{X})+$ oxaliplatin $(\mathrm{O})(\mathrm{XELOX})$ vs. infusional 5- FU/LV + O (FOLFOX-6) for metastatic colorectal cancer (MCRC). Proc Am Soc Clin Oncol 170s (abstr 4029)

Feliu J, Salud A, Escudero P et al (2006) XELOX (capecitabine plus oxaliplatin) as first-line treatment for elderly patients over 70 years of age with advanced colorectal cancer. Br J Cancer 94:969-975

Fischel J-L, Etienne M-C, Formento P, Milano G (1998) Search for the optimal schedule for the oxaliplatin/5-fluorouracil association modulated or not by folinic acid: preclinical data. Clin Cancer Res 4:2529-2535

Giacchetti S, Perpoint B, Zidani R et al (2000) Phase III multicenter randomized trial of oxaliplatin added to chronomodulated fluorouracil-leucovorin as first-line treatment of metastatic colorectal cancer. J Clin Oncol 18:136-147

Goldberg RM, Sargent DJ, Morton RF et al (2004) A randomized controlled trial of fluorouracil plus leucovorin, irinotecan, and oxaliplatin combinations in patients with previously untreated metastatic colorectal cancer. J Clin Oncol 22:23-30

Goldberg RM, Sargent DJ, Morton RF et al (2006) Randomized controlled trial of reduced-dose bolus fluorouracil plus leucovorin and irinotecan or infused fluorouracil plus leucovorin and oxaliplatin in patients with previously untreated metastatic colorectal cancer: a North American intergroup trial. J Clin Oncol 24:33473353

de Gramont A, Figer A, Seymour M et al (2000) Leucovorin and fluorouracil with or without oxaliplatin as first-line treatment in advanced colorectal cancer. J Clin Oncol 18:2938-2947

Grothey A, Jordan K, Kellner O et al. (2003): Capecitabine plus irinotecan (CAPIRI) vs capecitabine plus oxaliplatin (CAPOX) as first-line therapy of advanced colorectal cancer (ACRC): updated results of a randomized phase II trial. Proc ECCO 12, Eur J Cancer S90 (abstract \# 295)

Hochster HS, Hart LL, Ramanathan RK et al. (2006) Safety and efficacy of oxaliplatin/fluoropyrimidine regimens with or without bevacizumab as first-line treatment of metastatic colorectal cancer (mCRC): final analysis of the TREE-Study. Proc Am Soc Clin Oncol Annual Meeting 24, 18S (abstract 3510)

Kaplan ES, Meier P (1958) Non parametric estimation for incomplete observations. J Am Stat Assoc 53:557-580

Lévi F, Misset JL, Brienza S et al (1992) A chronopharmacologic phase II clinical trial with 5-fluorouracil, folinic acid, and oxaliplatin using ambulatory multichannel programmable pump. High antitumor effectiveness against metastatic colorectal cancer. Cancer 69:893-900

Liu G, Franssen E, Fitch MI, Warner E (1997) Patient preferences for oral versus intravenous palliative chemotherapy. J Clin Oncol 15:110-115

Machover D, Díaz-Rubio E, De Gramont A et al (1996) Two consecutive phase II study of oxaliplatin (L-OHP) for the treatment of patients with advanced colorectal carcinoma who were resistant to previous treatment with fluoropyrimidines. Ann Oncol 7:95-98

Mantel N (1966) Evaluation of survival data and two new ranks of order statistics arising in its considerations. Cancer Chemother Rep 50:163-170

Martoni AA, Pinto C, Di Fabio F et al (2006) Capecitabine plus oxaliplatin (XELOX) versus protracted 5-fluorouracil venous infusion plus oxaliplatin (pviFOX) as first-line treatment in advanced colorectal cancer: a GOAM phase II randomised study (FOCA trial). Eur J Cancer 42:3161-3168

Mayer RJ (2007) Should capecitabine replace infusional fluorouracil and leucovorin when combined with oxaliplatin in metastatic colorectal cancer? J Clin Oncol 25:4165-4167

Miller AB, Hoogstraten B, Staquet M, Winkler A (1981) Reporting results of cancer treatment. Cancer 47:207-214

Osoba D, Rodrigues G, Myles J, Zee B, Pater J (1998) Interpreting the significance of changes in health-related quality-of-Life scores. J Clin Oncol 16:139-144

Pfeiffer P, Mortensen JP, Bjerregaard B et al (2006) Patient preference for oral or intravenous chemotherapy: a randomised cross-over trial comparing capecitabine and Nordic fluorouracil/leucovorin in patients with colorectal cancer. Eur J Cancer 42:2738-2743

Porschen R, Arkenau H-T, Kubicka S et al (2007) Phase III study of capecitabine plus oxaliplatin compared with fluorouracil and leucovorin plus oxaliplatin in metastatic colorectal cancer: a final report of the AIO colorectal study group. J Clin Oncol 25:42174223

Raymond E, Chaney SG, Taamma A, Cvitkovic E (1998) Oxaliplatin: a review of preclinical and clinical studies. Ann Oncol 9:10531071

Salts LB, Clarke S, Diaz-Rubio E et al. (2007) Bevacizumab (Bev) in combination with XELOX or FOLFOX4: efficacy results from XELOX-1/NO16966, a randomized phase III trial in the first-line metastatic colorectal cancer (MCRC). Proc Am Soc Clin Oncol Gastrointestinal Cancer Symposium (abstract 238)

Sandomenico C, Filippelli G, Massidda B et al. (2006): Biweekly oxaliplatin plus Xeloda (OXXEL) versus oxaliplatin plus folinic acid-modulated 5-fluorouracil i.v. bolus (OXAFAFU) in metastatic colorectal carcinoma (MCC): safety interim analysis of a 
Southern Italy Cooperative oncology group (SICOG) phase III trial. Proc Am Soc Clin Oncol 168 s (abstract 3590)

Twelves C, Gollins S, Grieve R, Samuel L (2006) A randomised crossover trial comparing patient preference for oral capecitabine and 5-fluorouracil/leucovorin regimens in patients with advanced colorectal cancer. Ann Oncol 7:239-245
Ward SE, Kaltenthaler E, Cowan J et al (2006) The clinical and economic benefits of capecitabine and tegafur with uracil in metastatic colorectal cancer. Br J Cancer 95:27-33

Zeuli M, Nardoni C, Pino MS et al (2003) Phase II study of capecitabine and oxaliplatin as first-line treatment in advanced colorectal cancer. Ann Oncol 14:1378-1382 